\title{
Developmental stress affects song learning but not song complexity and vocal amplitude in zebra finches
}

\author{
Henrik Brumm • Sue Anne Zollinger • Peter J. B. Slater
}

Received: 17 November 2008 /Revised: 25 February 2009/Accepted: 7 March 2009/Published online: 24 March 2009

(C) The Author(s) 2009. This article is published with open access at Springerlink.com

\begin{abstract}
Several recent studies have tested the hypothesis that song quality in adult birds may reflect early developmental conditions, specifically nutritional stress during the nestling period. Whilst all of these earlier studies found apparent links between early nutritional stress and song quality, their results disagree as to which aspects of song learning or production were affected. In this study, we attempted to reconcile these apparently inconsistent results. Our study also provides the first assessment of song amplitude in relation to early developmental stress and as a potential cue to male quality. We used an experimental manipulation in which the seeds on which the birds were reared were mixed with husks, making them more difficult for the parents to obtain. Compared with controls, such chicks were lighter at fledging; they were thereafter placed on a normal diet and had caught up by 100 days. We show that nutritional stress during the first 30 days of life reduced the birds' accuracy of song syntax learning, resulting in poorer copies of tutor songs. Our experimental manipulations did not lead to significant changes in song amplitude, song duration or repertoire size. Thus, individual differences observed in song performance features probably reflect
\end{abstract}

Communicated by W.A. Searcy

H. Brumm $\cdot$ S. A. Zollinger $\cdot$ P. J. B. Slater

Institute of Behavioural and Neural Sciences, School of Biology,

University of St Andrews,

Fife, UK

Present Address:

H. Brumm $(\bowtie)$

Max Planck Institute for Ornithology,

Communication and Social Behaviour Group,

Eberhard-Gwinner-Str,

82319 Seewiesen, Germany

e-mail: brumm@orn.mpg.de differences in current condition or motivation rather than past condition.

Keywords Bird song · Condition-dependent signal ·

Early nutritional deficiency $\cdot$ Developmental stress

hypothesis · Song amplitude $\cdot$ Song learning ·

Taenopygia guttata $\cdot$ Zebra finch

In many bird species, females prefer males with complex songs, and it is thought that this preference evolved because song complexity reflects the quality of the male (Catchpole and Slater 2008). Sexual selection theory predicts that sexual traits indicating the sender's quality must be costly to develop or produce to preserve the honesty of the signal (Grafen 1990); otherwise, all individuals could produce these signals irrespective of their quality and the signal would become uninformative.

Which costs might constrain song complexity is a subject of debate (Gil and Gahr 2002). The nutritional stress hypothesis (Nowicki et al. 1998; Nowicki et al. 2002) proposes that juvenile condition may affect brain development and thus song learning skills. Only those males that are well fed during the nestling and fledgling period will have the resources to invest in optimal brain development, which enables them to learn complex songs. As a result, song complexity could function as an indicator of past condition. There are now several studies which have tested the nutritional stress hypothesis in the laboratory. Nowicki et al. (2002) found that food limitation in nestling swamp sparrows (Melospiza georgiana) led to a decrease in learning accuracy. Similarly, it has been shown that early nutritional stress in the European starling (Sturnus vulgaris) affected both repertoire size and the duration of adult songs (Buchanan et al. 2003). Moreover, several studies investi- 
gated stressors other than restricted nutrition (corticosterone administration, parasite infection and natal brood size) and reported effects on song control brain nuclei, song complexity and singing performance (Buchanan et al. 2003, 2004; Spencer et al. 2003, 2005; Soma et al. 2006). Therefore, the nutritional stress hypothesis should be considered as a particular case of a more general developmental stress hypothesis (Buchanan et al. 2003).

In zebra finches (Taenopygia guttata), there is inconsistent evidence on whether early nutritional stress impairs song learning and, if so, which song parameters may be affected. Spencer et al. (2003) used a method of food limitation developed by Lemon (1991), who showed that mixing the food seeds with husks reduces the food intake rate in zebra finches and, as a result, reduces the condition of the birds. Spencer et al. (2003) found that when they mixed food for nestlings with husk during the first 30 days of life, as adults, the birds had shorter song phrases and a smaller total syllable number than controls. However, these authors did not assess song learning, although an effect on this is one of the central predictions of the nutritional stress hypothesis. To date, only four studies have addressed whether early developmental stress causes differences in male song learning (Nowicki et al. 2002; Gil et al. 2006; Zann and Cash 2008; Holveck et al. 2008), but they have reported inconsistent results. Gil et al. (2006) manipulated the brood size of zebra finches to induce early developmental stress, but found no effect of the treatment on song duration, element repertoire or song rate. They also did not find a treatment effect on the syllable similarity between tutee and tutor songs as assessed by visual inspection of spectrograms. In an experiment with non-domesticated zebra finches raised with a seed-husk mixture, Zann and Cash (2008) showed that early nutritional condition did have an effect on adult song complexity. However, they found that stressed zebra finches developed longer songs, and did not find evidence for changes in repertoire size. Their study also failed to find an effect of early condition on learning accuracy. Most recently, Holveck et al. (2008) addressed the effect of developmental condition on song syntax learning and temporal song consistency in zebra finches using brood size manipulation to induce early developmental stress. They found that stressed birds do show evidence of impaired learning accuracy. There is thus no consensus on whether and how zebra finch song is an honest signal of developmental stress.

Whilst the focus of most experimental tests of the nutritional stress hypothesis has been on brain development, it is likely that other tissues such as muscles can be affected by poor early condition in nestling birds. The vocal control of bird song is based on a complex neuromuscular system involving the syrinx, respiratory system and upper vocal tract (Suthers et al. 1999), and therefore, deficiencies in muscular growth due to early nutritional stress may adversely affect song production. If such deficiencies are not compensated for later in life, then, in addition to song learning, the performance of songs may also be impaired by developmental stress. In particular, song amplitude is determined by the air pressure in the birds' air sacs, which is built up by the abdominal expiratory muscles (Plummer and Goller 2008). If the strength of the abdominal muscle sheets is permanently reduced by early developmental stress, then vocal amplitude could be a signal of past condition. The loudness of bird song is a performancerelated trait that can vary considerably between individuals (Brumm and Hultsch 2001; Brumm and Todt 2002; Brumm and Slater 2006; Brumm 2009), making it a possible candidate for a sexually selected signal component. However, the evolutionary consequences of this variation are only poorly understood (Gil and Gahr 2002).

In this study, we investigate whether nutritional stress early in life may affect the amplitude of adult songs and thus whether song intensity could be used as an honest signal in sexual selection by indicating a bird's past condition. Moreover, using the same food restriction method as in previous experiments, our study addresses the apparent contradictory findings regarding the effects of early developmental condition on song learning. Therefore, we investigated whether song learning is affected by early nutritional stress and which aspects of song structure may reflect the past condition of adult birds. If song complexity is indeed an honest signal of developmental stress, birds raised under a restricted food regime would be expected to develop less complex songs than birds with unlimited access to food.

\section{Materials and methods}

Subjects and housing

We used 12 adult zebra finch pairs for breeding. The females were between 10 and 11 months old, and all of them had been paired previously at an age of 110-140 days. During this previous breeding attempt, all females laid a clutch, but none reared any young because the clutches were taken from them as part of an experimental protocol. Between their first breeding attempt and the experiment reported here, male and female birds were kept separately in single-sex flocks ( 31 males and 20 females) in two aviaries $(1.7 \times 2.5$ and $2 \mathrm{~m}$ high $)$ which allowed auditory but no visual contact. In these aviaries, the birds were maintained on a 12:12-h light/dark schedule under fullspectrum lights, with air temperature ranging between $20^{\circ} \mathrm{C}$ and $24^{\circ} \mathrm{C}$. The finches were fed on an ad libitum diet of 
foreign finch mix (Haith's, Cleethorpes, UK) supplemented once a week with millet sprays and fresh greens. Fresh drinking water, oyster shell grit and cuttlebone were available ad libitum. All female birds were bred in our laboratory; the males were bred in our laboratory or at other UK universities.

Experimental manipulations and breeding procedure

Males and females were randomly paired and housed in cages $(128 \times 30$ and $40 \mathrm{~cm}$ high) equipped with nest boxes. Air temperature in these was $21-24^{\circ} \mathrm{C}$ and relative humidity was $40-50 \%$. To induce breeding, the pairs were provided with nesting material (hay and pieces of hemp string) and the light regime was shifted to $14: 10 \mathrm{~h}$ light/ dark. In addition, the cages were sprayed with water on three consecutive days to simulate rainfall. One pair failed to breed within 15 days and was removed from the experiment. Breeding pairs were fed on an ad libitum diet of foreign finch mix, and they were also given fresh greens (spinach, bean sprouts and dandelion leaves) and a protein supplement (Haith's egg biscuit) once a week. Fresh drinking water, oyster shell grit and cuttlebone were available ad libitum.

After hatching of the young, whole family groups were assigned to one of two experimental groups (STRESS and CONTROL groups) so that the average brood size for both groups was between 3.4 and 3.8 birds (later on, however, it became obvious that one brood from each experimental group contained only female chicks, so the actual sizes for broods with male chicks were two, three, four and five in the STRESS group and two, four, four, four and five in the CONTROL group). Following the protocol of Spencer et al. (2003), experimental manipulations began when broods were 5 days old and continued until 30 days of age, at which time the young reached nutritional independence. During this period, the CONTROL birds received their usual food as described above. The birds in the STRESS treatment also received fresh greens, protein supplement and foreign finch mix seeds ad libitum, but their seeds were mixed with seed husks in a seed/husk volume ratio of 1:3. Such mixing of seeds with husks has been shown to decrease the food intake rate of zebra finches and to make the birds increase their time spent foraging (Lemon 1991). Thus, this food manipulation will increase parental foraging effort and reduce the feeding rate of the young, simulating a poor resource environment.

All nestlings $(N=40)$ were weighed between 0900 and 1200 hours with an Ohaus Scout II digital precision balance (accuracy, $\pm 10 \mathrm{mg}$ ) every other day between day 5 and day 15 and then at regular intervals until day 30 . At 30 days, the food restriction was lifted, and thereafter, the STRESS birds also received ad libitum supplies of seeds without addi- tional husks. On day 35, the mothers were removed from the breeding cages, but the offspring were kept together with their fathers until days 60-65. This was done because interactions with a male tutor until this stage can affect song learning (Eales 1985; Slater et al. 1988). Between days 60 and 65 , all young were separated from their fathers and kept in sex-specific cages $(128 \times 30$ and $40 \mathrm{~cm}$ high). All males (and females, respectively) from one brood were housed together with the male (or female) young from another pair, resulting in flock sizes between three and five birds. One brood in each cage came from each of the two treatments. At day 100, by which time zebra finches usually reach maturity, and again at day 200, all birds were weighed and the length of their tarsi and wings measured. All chicks reached adulthood, except for one chick that died 1 day after hatching (i.e. before the assignment of experimental groups).

\section{Song recordings and analyses}

To quantify the fathers' (tutor) singing activity, each breeding male was recorded in his home cage on 3 days, which were evenly distributed over the food manipulation period (days 5-30). We used a Sennheiser ME 62 microphone and a Marantz PMD 670 solid-state recorder; recordings were made between 0900 and 1500 hours and lasted for $180 \mathrm{~min}$. Afterwards, we counted the number of song phrases produced by each male tutor and calculated a mean song rate per unit time.

At an age of 200-212 days, all male offspring $(N=18)$ were individually recorded in a wire cage $(25 \times 40$ and $30 \mathrm{~cm}$ high) with one single perch. The cage was placed in a separate, sound-shielded room. An omnidirectional microphone (Sennheiser ME 62) connected to a Marantz PMD 670 solid-state recorder was placed $50 \mathrm{~cm}$ above the perch to record the subject's vocalisations. This arrangement was chosen because bird song shows a directional sound radiation pattern (e.g. Brumm 2002), and by placing the microphone above the singing male, variation in sound level resulting from lateral head movements of the bird is minimised (Brumm and Todt 2002).

Birds vary the sound level of their songs with the intensity of the background noise (reviewed in Brumm and Slabbekoorn 2005) as well as the social context (Brumm and Todt 2004; Cynx and Gell 2004). Zebra finches have also been shown to adjust their vocal amplitude in relation to the communication distance (Brumm and Slater 2006). Therefore, it is crucial to take these parameters into account when trying to measure the sound levels of bird songs. To standardise the context and communication distance, all males were presented with a female, which they readily courted by producing so-called directed songs (Sossinka and Böhner 1980). Each male was presented with a 
different female, which was placed in a second cage $(33 \times$ 15 and $28 \mathrm{~cm}$ high) adjacent to the male's, resulting in a distance between the male and the female perch of $10 \mathrm{~cm}$. Thus, our recordings relate to courtship songs over a very short distance. In this context, females are likely to assess the amplitude of the male songs, and at the same time, sexual selection through female choice is most likely to play a role during courtship. Therefore, honesty of signalling may be of particular importance in the context studied in this experiment.

All digital audio recordings were made with a sampling frequency of $44.1 \mathrm{kHz}$ and 16-bit accuracy. For each male, we analysed ten song phrases and calculated mean values for each parameter. The digital sound files were transferred to a PC and the sound pressure level for each element of each song was measured following the procedure outlined by Brumm and Slater (2006) using Avisoft-SASLabPro 4.36 software (Avisoft Bioacoustics, Berlin, Germany). The software was calibrated as follows: A sine tone with constant amplitude was recorded with the same settings as used for the bird song recordings, and the sound level of the recorded tone was set to the value directly measured with a precision sound level metre (CEL 214, cel instruments, UK) at the position of the microphone. Prior to analysis, the song recordings were high-pass-filtered (cutoff frequency, $0.4 \mathrm{kHz}$; window type, Hamming; 1024 coefficients) to remove low-frequency noise produced by the ventilation system of the test room.

We also measured the duration of the song phrases and counted the total number of syllables per phrase. Previous studies on early developmental stress in zebra finches have looked at absolute syllable numbers to quantify song complexity (Zann and Cash 2008; Spencer et al. 2003). However, the relative syllable number in relation to the model song of the tutor rather than the absolute number of syllables may be a more accurate estimate of learning success. Therefore, we also calculated the percentage of copied syllables and the percentage of copied syllable types from the tutor song for each song phrase analysed.

In addition, we also calculated an overall similarity score between tutor and tutee songs using Sound Analysis Pro (Tchernichovski et al. 2000). This software is a powerful analysis tool to quantify the accuracy of song learning success in zebra finches (Tchernichovski et al. 2004). The similarity scores calculated by Sound Analysis Pro are not based on a single measure, but take three separate acoustic parameters into account: (1) the percentage of overall significant similarity between tutee and tutor song phrases, (2) the average fine-scale accuracy or matching of the acoustic features and (3) the sequential matching between tutee and tutor songs. For our analyses, we used the default settings of the Sound Analysis Pro similarity module. In laboratory populations of domesticated zebra finches, pairs of tutor-tutee songs usually show a similarity score above 60 , whereas similarity scores of 40 or less are found in random pairings where no imitation has occurred (Tchernichovski et al. 2000). In our data, the fathers' self-similarity score was 95 or higher.

\section{Statistical analysis}

We analysed our data using linear mixed models (SPSS v.15.0). The experimental treatment was declared as a fixed factor and the brood ID as random factor to control for the dependence of data from the same clutch. The tutor song rate and the brood size were included as covariates to control for possible effects on song learning of the nestling birds. Differences in tutor song rate were examined with $t$ tests.

\section{Results}

Nestling growth rates

Our food manipulation successfully induced early nutritional stress in the chicks of the STRESS group, as reflected in their lower growth rates (Fig. 1). At the start of the treatment (day 5), body mass was similar in both experimental groups, but after fledging (day 20), control birds were significantly heavier than the birds in the food manipulation group (LMM: $F_{1,38}=5.84, p=0.021$ ). This effect persisted when the fledglings reached independence around day 30 (LMM: $F_{1,38}=4.36, p=0.044$ ). By day 100 , however, the STRESS birds had caught up with the control group and their body mass was no longer significantly different (Table 1). Interestingly, the STRESS birds had shorter wings at day 100, more than 2 months after the food

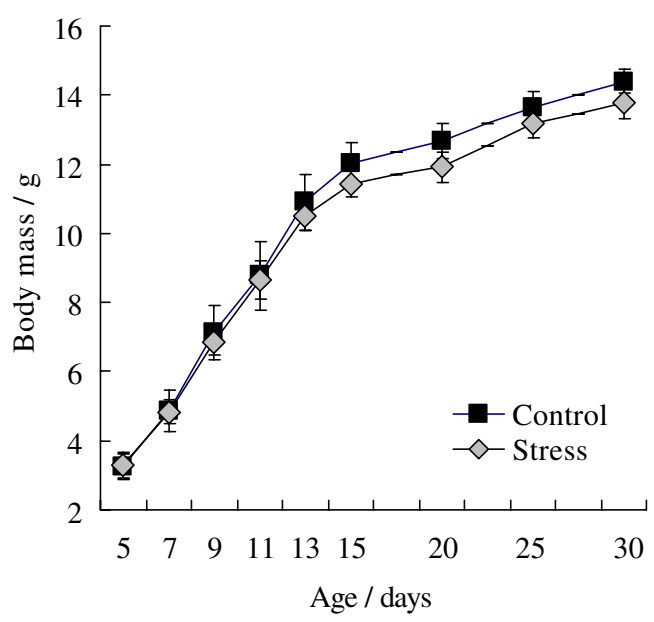

Fig. 1 Growth curves of nestling zebra finches $(m e a n \pm S D)$ experiencing food restriction (STRESS) and a control group (see "Materials and methods"). $N=20$ for each group 
Table 1 Results of linear mixed models testing the effect of the food restriction on body mass of zebra finches as well as measures of body size at day 100 and day 200 post-hatching

Birds of the STRESS group ( $N=8$ males and 12 females) were exposed to nutritional stress through food manipulation between days 5 and 30 ; the CONTROL birds ( $N=10$ males and 10 females) were raised under unrestricted foraging conditions (see "Materials and methods" for details).

\begin{tabular}{|c|c|c|c|c|c|}
\hline & \multicolumn{2}{|l|}{ Mean \pm SD } & \multirow[t]{2}{*}{$F$} & \multirow[t]{2}{*}{$d f$} & \multirow[t]{2}{*}{$p$} \\
\hline & STRESS & CONTROL & & & \\
\hline \multicolumn{6}{|l|}{ Males } \\
\hline \multicolumn{6}{|l|}{ Day 100} \\
\hline Mass (g) & $17.04 \pm 0.86$ & $17.87 \pm 2.88$ & 6.79 & $1,0.47$ & 0.517 \\
\hline Tarsus length (mm) & $16.7 \pm 0.6$ & $16.5 \pm 0.6$ & 1.01 & $1,7.60$ & 0.347 \\
\hline Wing length (mm) & $56.2 \pm 1.6$ & $58.7 \pm 1.2$ & 7.98 & $1,6.01$ & 0.030 \\
\hline \multicolumn{6}{|l|}{ Day 200} \\
\hline Mass (g) & $18.69 \pm 1.82$ & $18.80 \pm 3.22$ & 0.01 & 1,16 & 0.931 \\
\hline Tarsus length (mm) & $16.7 \pm 0.4$ & $16.6 \pm 0.5$ & 0.52 & 1,16 & 0.482 \\
\hline Wing length (mm) & $58.9 \pm 3.6$ & $60.5 \pm 4.6$ & 0.66 & $1,3.46$ & 0.469 \\
\hline \multicolumn{6}{|l|}{ Females } \\
\hline \multicolumn{6}{|l|}{ Day 100} \\
\hline Mass (g) & $17.19 \pm 1.56$ & $17.79 \pm 2.94$ & 0.53 & $1,8.12$ & 0.486 \\
\hline Tarsus length (mm) & $16.7 \pm 0.6$ & $16.3 \pm 0.9$ & 1.34 & $1,4.29$ & 0.307 \\
\hline Wing length (mm) & $56.6 \pm 2.2$ & $58.2 \pm 1.9$ & 3.04 & 1,20 & 0.097 \\
\hline \multicolumn{6}{|l|}{ Day 200} \\
\hline Mass (g) & $17.98 \pm 2.88$ & $18.43 \pm 2.39$ & 0.17 & $1,9.43$ & 0.691 \\
\hline Tarsus length (mm) & $16.7 \pm 0.5$ & $16.3 \pm 0.9$ & 1.35 & 1,20 & 0.259 \\
\hline Wing length (mm) & $57.8 \pm 1.3$ & $58.2 \pm 2.0$ & 0.08 & $1,6.98$ & 0.785 \\
\hline
\end{tabular}

restriction was lifted (but this difference was only significant in the males). This shows that despite the similar body mass, there was still a difference between the two treatment groups when birds reached maturity. By day 200, however, the stressed birds seemed to have fully compensated for the restriction, and no statistical difference in body size could be detected between the STRESS and CONTROL groups.

\section{Tutor song rates}

The food manipulation affected the activity budgets of the parents as well as the growth rates of the young (Fig. 2). Breeding control males sang on average more than four times as many song phrases per unit time than the males with the restricted food regime ( $t$-test: $\left.t_{9}=2.283, p=0.048\right)$.

Tutee song parameters

We analysed the songs of stressed and control males with regard to song structure and song performance, i.e. song

Fig. 2 Effect of food restriction on the singing rates of breeding zebra finches (STRESS: $N=5$ males, CONTROL: $N=6$ males)

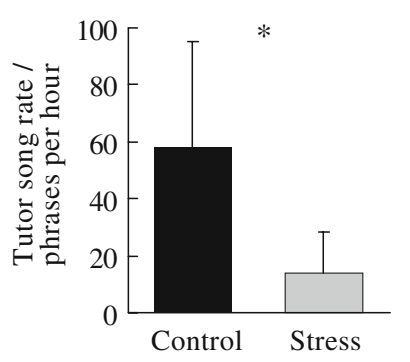

complexity and learning accuracy, and vocal amplitude. The duration of song phrases was not significantly affected by the experimental treatment (Fig. 3a; LMM: $F_{1,3.04}=0.56$, $p=0.509)$. STRESS and CONTROL males also did not differ significantly in the total number of song syllables $\left(F_{1,14}=1.52, p=0.238\right)$, the number of syllable types $\left(F_{1,5.12}=1.36, p=0.294\right)$ or the percentage of syllables copied from their respective tutors $\left(F_{1,5.52}=0.42, p=\right.$ 0.543; Fig. 3b-d). Stressed birds copied fewer syllable types from their fathers, but this difference was not statistically significant (Fig. 3e; $F_{1,5.55}=3.37, p=0.120$ ). On average, the control males had copied $95 \%$ of their syllable types from their tutors, whereas the STRESS birds copied only $70 \%$. Our treatment did have a statistically significant effect on the similarity score calculated by Sound Analysis Pro: STRESS birds scored lower than the control males (Fig. 3f; $F_{1,6.94}=12.47, p=0.010$; final model after excluding the non-significant covariates, i.e. tutor song rate and brood size), which indicates a lower learning accuracy in the birds that underwent early nutritional stress. This difference in song similarity was mainly due to the STRESS birds matching the sequencing of their fathers' songs less accurately $\left(F_{1,7.72}=7.35, p=0.028\right)$. The other two parameters used to calculate the similarity score, i.e. overall similarity $\left(F_{1,14}=1.76, p=0.205\right)$ and fine scale matching of acoustic features $\left(F_{1,5.26}=0.141, p=0.722\right)$, did not differ significantly between the two experimental groups. None of the measured song characteristics varied significantly with the tutor song rate $(F=0.01$ to $1.83, p=$ 
a

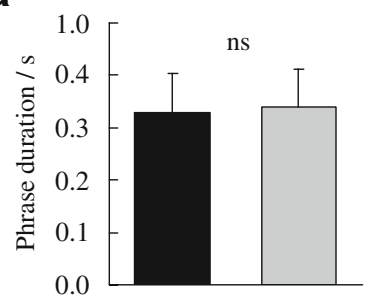

C

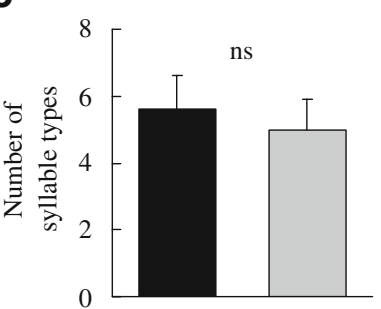

e

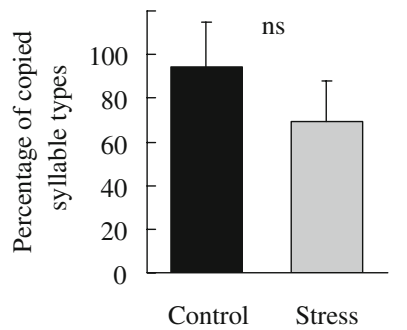

b

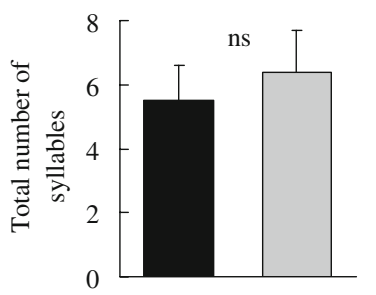

d

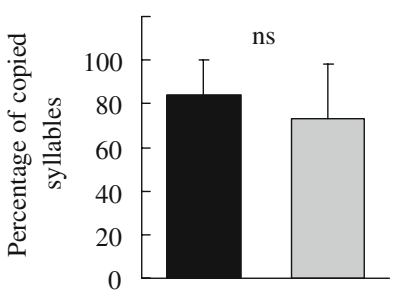

f

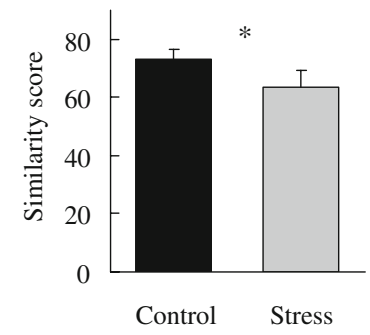

Fig. 3 Effects of nutritional stress between days 5 and 30 posthatching on the song structure in adult zebra finches. a Phrase duration, $\mathbf{b}$ total number of syllables, $\mathbf{c}$ number of syllables types, $\mathbf{d}$ percentage of syllables copied from the tutor, e percentage of syllable types copied from the tutor, $\mathbf{f}$ learning accuracy measured as mean similarity score

0.287 to 0.943$)$ or the brood size $(F=0.002$ to $1.66, p=$ 0.226 to 0.965$)$. There was a marked variation in vocal amplitude between males with a range of median song sound pressure levels of $15 \mathrm{~dB}$. However, there was no evidence that the experimental manipulation influenced any of our measures of song amplitude (Fig. 4; maximum song amplitude: $F_{1,14}=2.03, p=0.176$; median song amplitude: $F_{1,14}=1.47, p=0.245$; minimum song amplitude: $F_{1,14}=$ $1.60, p=0.266$ ).

\section{Discussion}

The results of our study support the idea that early nutritional stress impairs song learning, as measured by the song similarity scores between tutor and tutee. We found that food restriction during the sensitive period of song learning in zebra finches affected the accuracy of learning by the nestlings; in particular, birds fed suboptimally matched the sequencing of their fathers' songs less accurately than control males.

The experimental food manipulation not only led to slower growth and less accurate learning in food restricted nestlings but also affected the behaviour of the breeding parents. Our analysis of the fathers' singing activity showed that the breeding males that fed on manipulated food had a considerably decreased singing rate. This was most likely to be because the males in the STRESS treatment had to search longer for their food (Lemon 1991) and this time was found by a reduction in other activities, such as singing. Because the tutors in our study sang less, their young had fewer learning opportunities during their early sensorimotor period. However, despite their decreased singing activity, the tutor males in the food manipulation group still sang, on average, 14 song phrases per hour. With a photoperiod of 14-h light, this song rate will lead to almost 200 song phrases per day, which is more than sufficient for exact copying of song tutor phrases in zebra finches (Böhner 1983; Tchernichovski et al. 1999). Moreover, it has been shown that the sensitive period of song learning in zebra finches extends up to 65 days posthatching (Slater et al. 1988). The food restriction in our experiment was lifted after day 30, thus allowing an increase in singing performance and tutoring opportunity during the remaining sensorimotor period. Thus, although the tutor song rate differed significantly between the food restriction and the control group, it did not vary with any of the measured song parameters in the tutees. Reduced

Fig. 4 Effects of nutritional stress between days 5 and 30 post-hatching on the song performance in adult zebra finches. a Maximum song amplitude, b median song amplitude, c minimum song amplitude

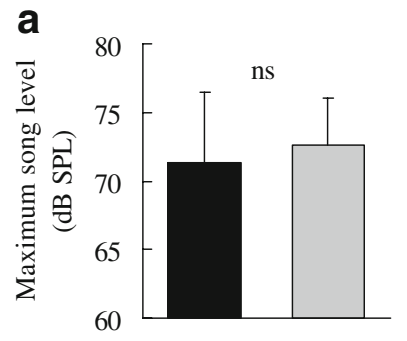

b
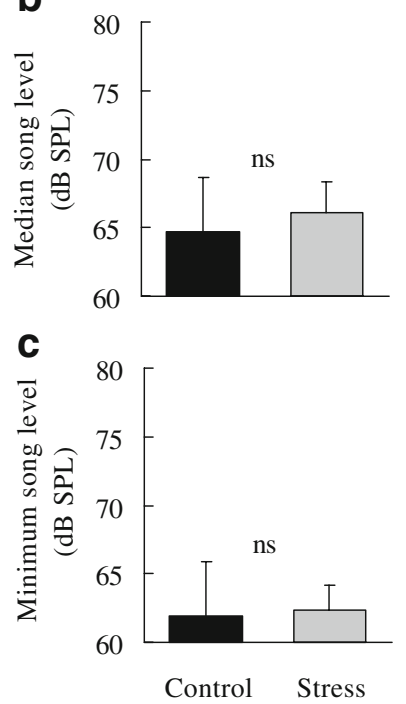
learning opportunities can therefore not explain the lower learning accuracy of the birds in our study.

We found that early nutritional stress lead to physical differences that persisted even after the birds reached sexual maturity. A similar result has been found by Arnold et al. (2007) who showed that female zebra finches that experienced early nutritional stress had shorter wings at day 100 . However, our results suggest that this effect is transient, as whilst the birds in our study did have shorter wings at day 100 , they had caught up with the control birds by day 200 . Thus, differences in wing length at day 100 seem rather to be an outcome of either delayed maturation or a temporary trade-off between skeletal/feather growth and other body traits. Such stress-induced differences in adult zebra finch biometry can have severe consequences for fitness, as they can be passed on to the next generation (Naguib and Gil 2005) and also affect the reproductive success of the offspring (Naguib et al. 2006).

The delayed growth in our birds indicates that our experimental protocol clearly affected the overall development of the young, and it is conceivable that this impact also extended to the development of the birds' brains. Evidence supporting this notion has been found by Buchanan et al. (2004) who looked at the effect of nutritional stress on the size of the song control nucleus HVC in zebra finches. However, a similar study could not find any connection between nutritional stress and brain development in zebra finches, with regard either to overall brain mass or to the volume of three different brain nuclei including HVC (Gil et al. 2006). Thus, the question of the mechanism that underlies the song deficiencies in stressed birds remains an open one. As our analysis controlled for tutor song rate, it is likely that the limited song learning success in our birds is an effect of stress-induced neural development, highlighting the poten- tial of song syntax to function as an honest signal of past condition.

Our finding that birds raised under the restricted food regime tended to copy fewer syllable types from their tutors is comparable, but not identical, to those in earlier studies that used the same method of food manipulation (Table 2). However, we did not find a difference in phrase duration between stressed and control birds as did the two earlier food restriction studies. On the other hand, we found that nutritional stress led to lower learning accuracy: Males raised under the restricted food regime developed poorer copies of their tutor's song. In particular, there was a poorer sequential match between tutor and tutee songs in the stressed birds. This finding is in line with the results of Holveck et al. (2008) who used brood size manipulation to induce developmental stress in young zebra finches (Table 2). They showed that birds from large broods, i.e. of poor early condition, copied the sequential order of song elements less accurately than birds from small broods. Interestingly, Holveck et al. (2008) and our study both obtained the same result regarding the sequential matching of tutor and tutee song as well as all other measures of song structure, although they used different methods for inducing developmental stress. Brood size manipulation varies both early nutrition and the social environment of the birds, whereas in our study, only nutritional condition was manipulated. Therefore, it is likely that the impaired learning accuracy in zebra finches found in both studies is a direct effect of nutritional stress rather than the social environment. This conclusion is also supported by the finding that the size of our experimental broods in the current study had no significant effect on any of the measured song parameters.

It appears that early developmental stress in zebra finches is reflected in adult song, but that the affected song

Table 2 Results of published studies on zebra finch song structure and song performance as an indicator of early developmental stress

\begin{tabular}{|c|c|c|c|c|c|c|c|c|}
\hline Study & $\begin{array}{l}\text { Method of } \\
\text { stress induction }\end{array}$ & $\begin{array}{l}\text { Phrase } \\
\text { duration }\end{array}$ & $\begin{array}{l}\text { Total } \\
\text { number of } \\
\text { syllables }\end{array}$ & $\begin{array}{l}\text { Total number } \\
\text { of different } \\
\text { syllable types }\end{array}$ & $\begin{array}{l}\text { Percentage } \\
\text { of copied } \\
\text { syllables }\end{array}$ & $\begin{array}{l}\text { Percentage } \\
\text { of copied } \\
\text { syllable types }\end{array}$ & $\begin{array}{l}\text { Learning } \\
\text { accuracy }\end{array}$ & $\begin{array}{l}\text { Song } \\
\text { amplitude }\end{array}$ \\
\hline $\begin{array}{l}\text { Spencer et al. } \\
2003\end{array}$ & Food restriction & Decreased & Reduced & No effect & & & & \\
\hline Gil et al. 2006 & $\begin{array}{l}\text { Brood size } \\
\text { manipulation }\end{array}$ & No effect & No effect & No effect & & & No effect ${ }^{a}$ & \\
\hline $\begin{array}{l}\text { Zann and Cash } \\
2008\end{array}$ & Food restriction & Increased & No effect & No effect & & & No effect ${ }^{b}$ & \\
\hline $\begin{array}{l}\text { Holveck et al. } \\
2008\end{array}$ & $\begin{array}{l}\text { Brood size } \\
\text { manipulation }\end{array}$ & No effect & No effect & No effect & No effect & No effect & Decreased $^{\mathrm{c}}$ & \\
\hline This study & Food restriction & No effect & No effect & No effect & No effect & No effect & Decreased $^{\mathrm{b}}$ & No effect \\
\hline
\end{tabular}

Cells with no entries indicate that the respective trait has not been investigated

${ }^{\text {a }}$ Based on visual comparisons of tutor and tutee songs

${ }^{\mathrm{b}}$ Based on spectral parameter analysis with Sound Analysis Pro software

${ }^{\mathrm{c}}$ Based on spectral parameter analysis with Luscinia software 
parameters differ between studies. However, when Gil et al. (2006) manipulated brood sizes to induce nutritional stress, they did not find an effect on phrase duration, syllable number or learning accuracy between groups despite having a much larger sample size than in any other study, including ours. However, evidence from a different species, the swamp sparrow, indicates that learning accuracy can indeed be affected by early nutritional stress (Nowicki et al. 2002). This notion is corroborated by our findings as well as those by Holveck et al. (2008).

In addition to structural parameters that have been examined in earlier studies, we also investigated the song performance in terms of vocal amplitude. In contrast to our findings on learning accuracy, we found no evidence that song amplitude was affected by our experimental manipulations. This means that nutritional stress early in life either had no influence on adult song intensity or that an effect was masked by other influences, such as motivation. However, we do not think the latter is very likely because of the very standardised experimental conditions in our study. Also, all males were tested in the same context (i.e. courtship), and the adult males in our two experimental groups had a similar size and body mass. Therefore, we conclude that vocal amplitude of adult song is probably not an indicator of early nutritional stress. Several studies show that adult birds differ markedly in their song amplitude (Brumm and Hultsch 2001; Brumm and Todt 2002; Brumm and Slater 2006; Brumm 2009). Our study suggests that variation in performance may reflect individual differences in current condition or motivation rather than past condition.

By and large, the majority of studies on early developmental stress in zebra finches indicate that song complexity, measured as song duration or repertoire size, is not affected by early condition. However, single studies yielded contrasting results, which might be due to differences in experimental protocols and data analysis (Table 2). In conclusion, our findings confirm that food manipulation (mixing seeds with husks) is a useful technique for inducing early developmental stress in zebra finches. Moreover, we found that such food restriction affects learning accuracy, resulting in poorer song copies in adult birds. However, it remains to be shown whether song syntax in zebra finches functions as an honest signal of male quality. At present, it is unclear whether females will detect the altered sequencing of song elements and whether they use this information to assess the past condition of a male.

Acknowledgements We thank Stefan Leitner and two anonymous referees for helpful comments on the manuscript. Financial support was provided by the BBSRC (award BB/E003494/1) and the German Research Foundation (Emmy Noether fellowship to H.B., award BR 2309/6-1). The experiment described in this study complies with the current laws of Scotland.
Open Access This article is distributed under the terms of the Creative Commons Attribution Noncommercial License which permits any noncommercial use, distribution, and reproduction in any medium, provided the original author(s) and source are credited.

\section{References}

Arnold KE, Blount JD, Metcalfe NB, Orr KJ, Adam A, Houston D, Monaghan P (2007) Sex-specific differences in compensation for poor neonatal nutrition in the zebra finch Taeniopygia guttata. J Avian Biol 38:356-366

Böhner J (1983) Song learning in the zebra finch (Taeniopygia guttata): selectivity in the choice of a tutor and accuracy of song copies. Anim Behav 31:231-237

Brumm H (2002) Sound radiation patterns in nightingale (Luscinia megarhynchos) songs. J Ornithol 143:468-471

Brumm H (2009) Song amplitude and body size in birds. Behav Ecol Sociobiol (in press). doi:10.1007/s00265-009-0743-4

Brumm H, Hultsch H (2001) Pattern amplitude is related to pattern imitation during the song development of nightingales. Anim Behav 61:747-754

Brumm H, Slabbekoorn H (2005) Acoustic communication in noise. Adv Study Behav 35:151-209

Brumm H, Slater PJB (2006) Animals can vary signal amplitude with receiver distance: evidence from zebra finch song. Anim Behav 72:699-705

Brumm H, Todt D (2002) Noise-dependent song amplitude regulation in a territorial songbird. Anim Behav 63:891-897

Brumm H, Todt D (2004) Male-male vocal interactions and the adjustment of song amplitude in a territorial bird. Anim Behav 67:281-286

Buchanan KL, Spencer KA, Goldsmith AR, Catchpole CK (2003) Song as an honest signal of past developmental stress in the European starling (Sturnus vulgaris). Proc R Soc Lond Ser B 270:1149-1156

Buchanan KL, Leitner S, Spencer KA, Goldsmith AR, Catchpole CK (2004) Developmental stress selectively affects the song control nucleus HVC in the zebra finch. Proc R Soc Lond B 271:2381-2386

Catchpole CK, Slater PJB (2008) Bird song biological themes and variations, 2nd edn. Cambridge University Press, Cambridge

Cynx J, Gell C (2004) Social mediation of vocal amplitude in a songbird Taeniopygia guttata. Anim Behav 67:451-455

Eales LA (1985) Song learning in zebra finches: some effects of song model availability on what is learnt and when. Anim Behav 33:1293-1300

Gil D, Gahr M (2002) The honesty of bird song: multiple constraints for multiple traits. Trends Ecol Evol 17:133-141

Gil D, Naguib M, Riebel K, Rutstein A, Gahr M (2006) Early condition song learning and the volume of song brain nuclei in the zebra finch (Taeniopygia guttata). J Neurobiol 66:1602-1612

Grafen A (1990) Biological signals as handicaps. J Theor Biol 144:517-546

Holveck M-J, Vieira de Castro AC, Lachlan RF, ten Cate C, Riebel K (2008) Accuracy of song syntax learning and singing consistency signal early condition in zebra finches. Behav Ecol 19:1267-1281

Lemon WC (1991) Fitness consequences of foraging behaviour in the zebra finch. Nature 352:153-155

Naguib M, Gil D (2005) Transgenerational effects on body size caused by early developmental stress in zebra finches. Biol Lett 1:95-97

Naguib M, Nemitz A, Gil D (2006) Maternal developmental stress reduces reproductive success of female offspring in zebra finches. Proc R Soc Lond Ser B 273:1901-1905

Nowicki S, Peters S, Podos J (1998) Song learning early nutrition and sexual selection in songbirds. Am Zool 38:179-190 
Nowicki S, Searcy WA, Peters S (2002) Brain development song learning and mate choice in birds: a review and experimental test of the "nutritional stress hypothesis". J Comp Physiol 188:1003-1014

Plummer EM, Goller F (2008) Singing with reduced air sac volume causes uniform decrease in airflow and sound amplitude in the zebra finch. J Exp Biol 211:66-78

Slater PJB, Eales LA, Clayton NS (1988) Song learning in zebra finches: progress and prospects. Adv Study Behav 18:1-34

Soma M, Takahasi M, Ikebuchi M, Yamada H, Suzuki M, Hasegawa T, Okanoya K (2006) Early rearing conditions affect the development of body size and song in Bengalese finches. Ethology 112:10711078

Sossinka R, Böhner J (1980) Song types in the zebra finch. Zeitschrift für Tierpsychologie 53:123-132

Spencer KA, Buchanan KL, Goldsmith AR, Catchpole CK (2003) Song as an honest indicator of developmental stress in the zebra finch (Taeniopygia guttata). Horm Behav 44:132-139
Spencer KA, Buchanan KL, Leitner S, Goldsmith AR, Catchpole CK (2005) Parasites affect song complexity and neural development in a songbird. Proc R Soc Lond Ser B 272:2037-2043

Suthers RA, Goller F, Pytte C (1999) The neuromuscular control of bird song. Philos Trans R Soc Lond Ser B 354:927-939

Tchernichovski O, Lints TJ, Mitra PP, Nottebohm F (1999) Vocal imitation in zebra finches is inversely related to model abundance. Proc Natl Acad Sci U S A 96:12901-12904

Tchernichovski O, Nottebohm F, Ho CE, Pesaran B, Mitra PP (2000) A procedure for automated measurement of song similarity. Anim Behav 59:1167-1176

Tchernichovski O, Lints TJ, Derégnaucourt S, Cimenser A, Mitra PP (2004) Studying the song development process: rationale and methods. Ann NY Acad Sci 1016:348-363

Zann R, Cash E (2008) Developmental stress impairs song complexity but not learning accuracy in non-domesticated zebra finches (Taeniopygia guttata). Behav Ecol Sociobiol 62:391-400 